CASE STUDY

\title{
CHANGING ROLE OF OCCUPATIONAL THERAPIST: TO BRING STROKE PATIENT BACK TO WORK.
}

\begin{abstract}
The growing incidence of stroke is one of the major causes of disability in Pakistan leading to restriction in participation and functional occupation of every day task, return to previous role of life and meeting social economical challenges. Many of the studies have revealed that early intervention of rehabilitative approach may assist the early comeback of the patient to work. In Pakistan occupational therapist plays dynamic role that engrosses combination of roles of Occupational Therapist for reintegration of patient in the society.
\end{abstract}

The aim of this study is to promote the understanding of role adaptation by therapist based on patient's occupational need in context of functional restoration, from counselor to advocator, social worker and vocational rehabilitator. Therapist switched roles for the reintegration of 24 year- old man with post stroke functional independence measurement ( FIM) score 2, ( completely dependent ) Initially intervention plan was proposed for six months based on COOP (cognitive orientation to daily occupational performance) along with compensatory and rehabilitative approach, due to relapse it extended for one and a half year.

The FIM scores was monitored independence measurement in occupational performance that was gained from 2 to 5 (mild assistance level) and the role support was provided accordingly.

In conclusion, the FIM scores improvement needs the interchangeable role of the therapist for promotion and improvement of occupational performance to restore role of client for back to work.

\section{KEYWORDS}

FIM, COOP, IFRA, Stroke, Disability, Reintegration, Psychodynamic

\author{
Neelum Zehra Bukhari \\ HOD Occupational Therapy \\ Ziauddin College of Rehabilitation sciences \\ Ziauddin University \\ neelum.zehra@zu.edu.pk
}

[Bukhari NZ. Changing Role of Occupational Therapist: To Bring Stroke Patient Back to Work. Pak. j. rehabil. 2018;7(1):54-56] 


\section{INTRODUCTION}

Stroke is one of the leading causes affecting the ability of people to regain completely or near to their previous functional status. It is considered as the third most common cause of death and the first reason of disability in developing and developed country'. Though in west it is getting decline but at south Asian countries, it has increased and is predicted to rise ${ }^{2}$. It is a harsh reality that Pakistani community has an unwelcoming attitude towards disability and built in environment does not accommodate the disables to show their abilities or get involved with their limited abilities ${ }^{3}$. The role of Occupational therapist can be adapted to improve the participation of stroke patient in the community once again. Since there is lack of statistical data but an estimated numbers through hospitals rehabs department and national body of Occupational therapy around 150 Occupational Therapist in the country and 95 percent of them work in pediatric section where as only $5 \%$ work at acute care occupational therapy. The role of occupational therapist is limited to Activities of Daily Living (ADL) recovery and maintenance of skills. There is very less focus on the restoration of employment and social reintegration 4 . Moreover after the acute care occupational therapy the follow-up or the continuation of the therapy is almost less than $2 \%$. Even if the therapist would plan to work on back to work, it is not possible due to lack of awareness of professional expertise. Many studies in West have shown the remarkable results of multidisciplinary team effort for stroke rehabilitation that involve from Daily activities to social reintegration ${ }^{5}$.

\section{CASE REVIEW}

Case Review of a young boy having left side paralysis, under the multiple role of occupational therapist got an opportunity to reintegrate in community.

Mr. D was taken to stroke unit from the emergency department of a renowned tertiary care hospital. The acute care occupational therapy (ACOT) assessed him on initial assessment for functional restoration (IFRA) and functional independence measurement (FIM) that scored impaired ADL, communication Skills and muscle endurance and scored 2 that is complete assistance required for functional activities. During the hospitalization, initially his family was guided for positioning and the duration for different positions since frequency of change has significant impact on circulation and neuromuscular health. Mr. D Being young patient was quite impatient and eager to get back to his previous life style. It was very difficult to make him comprehendible regarding his health seriousness. The poor understanding of family regarding stroke and its consequences was another challenge for the therapist to train Mr. D.

The first step was to enroll the family for the stroke support group to promote awareness and acceptance of the condition. Secondly, compensatory strategies were needed, as Mr. D strength was his right hand so, one handed techniques for position changing and maintenance of position was promoted with mild assistance. Rehabilitative frame work was selected for his further ADL promotion that enhances his FIM score from 2 to 4 that is moderate to mild assistance. It made him capable to dress himself with partial assistance and motivated him to work more independently. It not only introduced hope in the Patient for previous life role but also encourage the bilateral use of hand. Along with other rehabilitation team members (physiotherapist) work on his standing, shifting to wheel chair and weight transferring from one limb to another was list down in the goal. After 15 days at acute care he was discharge with the safety home discharge plan along with customized therapeutic plan and recommended some assistive devices. Later he was asked to follow as outpatient. Till here the acute care Occupational Therapist focused on the early basic functional restoration. For the Departmental therapy the role of Occupational therapist changed into a motivational speaker, social worker and a vocational trainer.

It was not easy for Mr. D to bear the expense of the rehabilitation; he had lost his job with no medical compensation from his work. He belongs to a lower middle class and already in debt with admission expenses. Now the occupational therapist moved in to the role of social worker and looked for the welfare policy and sponsor for his therapies, through different process of the applications and opportunities finally Mr. D was able to get discount for the services of rehabilitation. The rehabilitative frame of reference with the combination of psychodynamic frame work was selected for Mr. D social reintegration. The treatment modalities were not just the active passive tools of occupational therapy but counseling of family, cognitive therapy and diversion therapy for him was also inculcated to have a restart. AS a role of social worker, arrangement for orthotic, knee gaiter and wheel chair was made. The short term goal was to minimize the assistance and promote assistive walking. At the same time search for a suitable job as per his capabilities was in process.

Though it was very difficult and beyond the job responsibility but the goal of social reintegration and back to work is an occupational therapy domain. Finally Mr. D started assistive shifting and slight assistive walk, all this journey from maximum to mild assistance took seven months.

The whole prognosis twisted when Mr. D suffered from another hemorrhage, it reversed his condition. 
This episode of CVA shattered him and his family lost all the hope. The only light at the end of the tunnel was occupational therapy that still focused to improve the quality of life. It was more than a year now and the functional empowerment that was regained was lost. He took rest of three months and we started the rehab process once again.

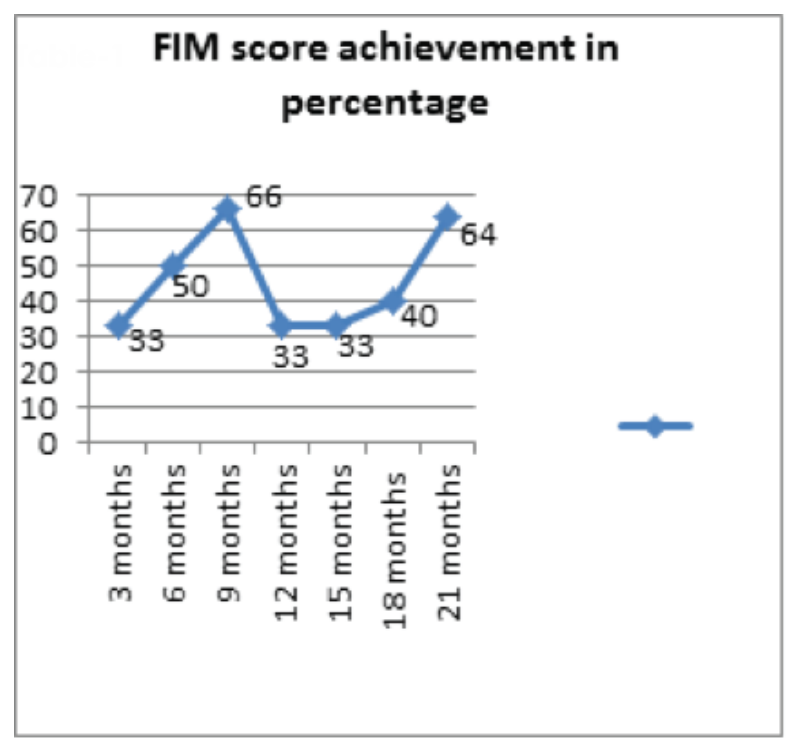

\section{Graph-1}

The FIM score was 2 (graph-1), with spasticity, more slurred speech and very poor work tolerance, the only strength was Mr. D motivation. Once again the journey started, this time the goal was to promote work tolerance and set realistic goals for getting back to work. After the continuous therapies, support group sessions and vocational training of six month, we were able to get a suitable job for him. This job demanded sitting tolerance and updating the appointments of clients at the consultant clinic. Mr. D still follows his therapy plan for maintenance and goes for his work daily. He has friends, every day task and a reason to work for himself and his family. His limitation has not set him apart from the community and work.

\section{CONCLUSION}

Improvement in Functional independence requires interchangeable role of the therapist to restore occupational performance till the patient reintegrate back to work or previous life role.

\section{REFERENCES}

[1] Hillis AE, Tippett DC. Stroke recovery: Surprising influences and residual consequences. Adv Med. 2014 doi: [10.1155/2014/378263]

[2] Khealani BA, Hameed B, Mapari UU. Stroke in Pakistan. J. Pak. Med. Assoc. 2008;58(7):400-3 $\mathrm{h}$ t t p s : / / pd f s. se man t i c s c hol ar.org/ae4d/9b44400c095543482ce527053d702 c534159.pdf

[3] Rathore FA, New PW, Iftikhar A. A report on disability and rehabilitation medicine in Pakistan: past, present, and future directions. Arch Phys Med Rehab. 2011;92(1):161-6.

[4] Cancelliere C, Donovan J, Stochkendahl MJ, Biscardi M, Ammendolia C, Myburgh C et al. Factors affecting return to work after injury or illness: best evidence synthesis of systematic reviews. Chiropr \& Manual Ther. 2016;24(1):32.

[5] Vestling $M$, Tufvesson B, Iwarsson S. Indicators for return to work after stroke and the importance of work for subjective well-being and life satisfaction. J Rehabil Med. 2003;35(3):127-31 